\title{
Supervised Grammar Induction using Training Data with Limited Constituent Information *
}

\author{
Rebecca Hwa \\ Division of Engineering and Applied Sciences \\ Harvard University \\ Cambridge, MA 02138 USA \\ rebecca@eecs.harvard.edu
}

\begin{abstract}
Corpus-based grammar induction generally relies on hand-parsed training data to learn the structure of the language. Unfortunately, the cost of building large annotated corpora is prohibitively expensive. This work aims to improve the induction strategy when there are few labels in the training data. We show that the most informative linguistic constituents are the higher nodes in the parse trees, typically denoting complex noun phrases and sentential clauses. They account for only $20 \%$ of all constituents. For inducing grammars from sparsely labeled training data (e.g., only higher-level constituent labels), we propose an adaptation strategy, which produces grammars that parse almost as well as grammars induced from fully labeled corpora. Our results suggest that for a partial parser to replace human annotators, it must be able to automatically extract higher-level constituents rather than base noun phrases.
\end{abstract}

\section{Introduction}

The availability of large hand-parsed corpora such as the Penn Treebank Project has made high-quality statistical parsers possible. However, the parsers risk becoming too tailored to these labeled training data that they cannot reliably process sentences from an arbitrary domain. Thus, while a parser trained on the Wall Street Journal corpus can fairly accurately parse a new Wall Street Journal article, it may not perform as well on a New Yorker article. To parse sentences from a new domain, one would normally directly induce a new grammar

* This material is based upon work supported by the National Science Foundation under Grant No. IRI 9712068. We thank Stuart Shieber for his guidance, and Lillian Lee, Ric Crabbe, and the three anonymous reviewers for their comments on the paper. from that domain, in which the training process would require hand-parsed sentences from the new domain. Because parsing a large corpus by hand is a labor-intensive task, it would be beneficial to minimize the number of labels needed to induce the new grammar.

We propose to adapt a grammar already trained on an old domain to the new domain. Adaptation can exploit the structural similarity between the two domains so that fewer labeled data might be needed to update the grammar to reflect the structure of the new domain. This paper presents a quantitative study comparing direct induction and adaptation under different training conditions. Our goal is to understand the effect of the amounts and types of labeled data on the training process for both induction strategies. For example, how much training data need to be hand-labeled? Must the parse trees for each sentence be fully specified? Are some linguistic constituents in the parse more informative than others?

To answer these questions, we have performed experiments that compare the parsing qualities of grammars induced under different training conditions using both adaptation and direct induction. We vary the number of labeled brackets and the linguistic classes of the labeled brackets. The study is conducted on both a simple Air Travel Information System (ATIS) corpus (Hemphill et al., 1990) and the more complex Wall Street Journal (WSJ) corpus (Marcus et al., 1993).

Our results show that the training examples do not need to be fully parsed for either strategy, but adaptation produces better grammars than direct induction under the conditions of minimally labeled training data. For instance, the most informative brackets, which label constituents higher up in the parse trees, typically 
identifying complex noun phrases and sentential clauses, account for only $17 \%$ of all constituents in ATIS and $21 \%$ in WSJ. Trained on this type of label, the adapted grammars parse better than the directly induced grammars and almost as well as those trained on fully labeled data. Training on ATIS sentences labeled with higher-level constituent brackets, a directly induced grammar parses test sentences with $66 \%$ accuracy, whereas an adapted grammar parses with $91 \%$ accuracy, which is only $2 \%$ lower than the score of a grammar induced from fully labeled training data. Training on WSJ sentences labeled with higher-level constituent brackets, a directly induced grammar parses with $70 \%$ accuracy, whereas an adapted grammar parses with $72 \%$ accuracy, which is $6 \%$ lower than the score of a grammar induced from fully labeled training data.

That the most informative brackets are higher-level constituents and make up only onefifth of all the labels in the corpus has two implications. First, it shows that there is potential reduction of labor for the human annotators. Although the annotator still must process an entire sentence mentally, the task of identifying higher-level structures such as sentential clauses and complex nouns should be less tedious than to fully specify the complete parse tree for each sentence. Second, one might speculate the possibilities of replacing human supervision altogether with a partial parser that locates constituent chunks within a sentence. However, as our results indicate that the most informative constituents are higher-level phrases, the parser would have to identify sentential clauses and complex noun phrases rather than low-level base noun phrases.

\section{Related Work on Grammar Induction}

Grammar induction is the process of inferring the structure of a language by learning from example sentences drawn from the language. The degree of difficulty in this task depends on three factors. First, it depends on the amount of supervision provided. Charniak (1996), for instance, has shown that a grammar can be easily constructed when the examples are fully labeled parse trees. On the other hand, if the examples consist of raw sentences with no extra struc- tural information, grammar induction is very difficult, even theoretically impossible (Gold, 1967). One could take a greedy approach such as the well-known Inside-Outside re-estimation algorithm (Baker, 1979), which induces locally optimal grammars by iteratively improving the parameters of the grammar so that the entropy of the training data is minimized. In practice, however, when trained on unmarked data, the algorithm tends to converge on poor grammar models. For even a moderately complex domain such as the ATIS corpus, a grammar trained on data with constituent bracketing information produces much better parses than one trained on completely unmarked raw data (Pereira and Schabes, 1992). Part of our work explores the in-between case, when only some constituent labels are available. Section 3 defines the different types of annotation we examine.

Second, as supervision decreases, the learning process relies more on search. The success of the induction depends on the initial parameters of the grammar because a local search strategy may converge to a local minimum. For finding a good initial parameter set, Lari and Young (1990) suggested first estimating the probabilities with a set of regular grammar rules. Their experiments, however, indicated that the main benefit from this type of pretraining is one of run-time efficiency; the improvement in the quality of the induced grammar was minimal. Briscoe and Waegner (1992) argued that one should first hand-design the grammar to encode some linguistic notions and then use the reestimation procedure to fine-tune the parameters, substituting the cost of hand-labeled training data with that of hand-coded grammar. Our idea of grammar adaptation can be seen as a form of initialization. It attempts to seed the grammar in a favorable search space by first training it with data from an existing corpus. Section 4 discusses the induction strategies in more detail.

A third factor that affects the learning process is the complexity of the data. In their study of parsing the WSJ, Schabes et al. (1993) have shown that a grammar trained on the InsideOutside re-estimation algorithm can perform quite well on short simple sentences but falters as the sentence length increases. To take this factor into account, we perform our experiments 


\begin{tabular}{|l|l|c|c|}
\hline Categories & Labeled Sentence & ATIS & WSJ \\
\hline \hline HighP & (I want (to take (the flight with at most one stop))) & $17 \%$ & $21 \%$ \\
\hline BaseNP & (I) want to take (the flight) with (at most one stop) & $27 \%$ & $29 \%$ \\
\hline BaseP & (I) want to take (the flight) with (at most one) stop & $32 \%$ & $30 \%$ \\
\hline AllNP & (I) want to take ((the flight) with (at most one stop)) & $37 \%$ & $43 \%$ \\
\hline NotBaseP & (I (want (to (take (the flight (with (at most one stop))))))) & $68 \%$ & $70 \%$ \\
\hline
\end{tabular}

Table 1: The second column shows how the example sentence $((I)$ (want (to (take ((the flight) (with ((at most one) stop $))))))$ ) is labeled under each category. The third and fourth columns list the percentage break-down of brackets in each category for ATIS and WSJ respectively.

on both a simple domain (ATIS) and a complex one (WSJ). In Section 5, we describe the experiments and report the results.

\section{Training Data Annotation}

The training sets are annotated in multiple ways, falling into two categories. First, we construct training sets annotated with random subsets of constituents consisting $0 \%, 25 \%, 50 \%$, $75 \%$ and $100 \%$ of the brackets in the fully annotated corpus. Second, we construct sets training in which only a certain type of constituent is annotated. We study five linguistic categories. Table 1 summarizes the annotation differences between the five classes and lists the percentage of brackets in each class with respect to the total number of constituents ${ }^{1}$ for ATIS and WSJ. In an AllNP training set, all and only the noun phrases in the sentences are labeled. For the BaseNP class, we label only simple noun phrases that contain no embedded noun phrases. Similarly for a BaseP set, all simple phrases made up of only lexical items are labeled. Although there is a high intersection between the set of BaseP labels and the set of BaseNP labels, the two classes are not identical. A BaseNP may contain a BaseP. For the example in Table 1, the phrase "at most one stop" is a BaseNP that contains a quantifier BaseP "at most one." NotBaseP is the complement of BaseP. The majority of the constituents in a sentence belongs to this category, in which at least one of the constituent's sub-constituents is not a simple lexical item. Finally, in a HighP set, we label only complex phrases that decom-

\footnotetext{
${ }^{1}$ For computing the percentage of brackets, the outermost bracket around the entire sentence and the brackets around singleton phrases (e.g., the pronoun "I" as a BaseNP) are excluded because they do not contribute to the pruning of parses.
}

pose into sub-phrases that may be either another HighP or a BaseP. That is, a HighP constituent does not directly subsume any lexical word. A typical HighP is a sentential clause or a complex noun phrase. The example sentence in Table 1 contains 3 HighP constituents: a complex noun phrase made up of a BaseNP and a prepositional phrase; a sentential clause with an omitted subject NP; and the full sentence.

\section{Induction Strategies}

To induce a grammar from the sparsely bracketed training data previously described, we use a variant of the Inside-Outside re-estimation algorithm proposed by Pereira and Schabes (1992). The inferred grammars are represented in the Probabilistic Lexicalized Tree Insertion Grammar (PLTIG) formalism (Schabes and Waters, 1993; Hwa, 1998a), which is lexicalized and context-free equivalent. We favor the PLTIG representation for two reasons. First, it is amenable to the Inside-Outside re-estimation algorithm (the equations calculating the inside and outside probabilities for PLTIGs can be found in Hwa (1998b)). Second, its lexicalized representation makes the training process more efficient than a traditional PCFG while maintaining comparable parsing qualities.

Two training strategies are considered: direct induction, in which a grammar is induced from scratch, learning from only the sparsely labeled training data; and adaptation, a two-stage learning process that first uses direct induction to train the grammar on an existing fully labeled corpus before retraining it on the new corpus. During the retraining phase, the probabilities of the grammars are re-estimated based on the new training data. We expect the adaptive method to induce better grammars than direct induction when the new corpus is only partially 
annotated because the adapted grammars have collected better statistics from the fully labeled data of another corpus.

\section{Experiments and Results}

We perform two experiments. The first uses ATIS as the corpus from which the different types of partially labeled training sets are generated. Both induction strategies train from these data, but the adaptive strategy pretrains its grammars with fully labeled data drawn from the WSJ corpus. The trained grammars are scored on their parsing abilities on unseen ATIS test sets. We use the non-crossing bracket measurement as the parsing metric. This experiment will show whether annotations of a particular linguistic category may be more useful for training grammars than others. It will also indicate the comparative merits of the two induction strategies trained on data annotated with these linguistic categories. However, pretraining on the much more complex WSJ corpus may be too much of an advantage for the adaptive strategy. Therefore, we reverse the roles of the corpus in the second experiment. The partially labeled data are from the WSJ corpus, and the adaptive strategy is pretrained on fully labeled ATIS data. In both cases, part-of-speech(POS) tags are used as the lexical items of the sentences. Backing off to POS tags is necessary because the tags provide a considerable intersection in the vocabulary sets of the two corpora.

\subsection{Experiment 1: Learning ATIS}

The easier learning task is to induce grammars to parse ATIS sentences. The ATIS corpus consists of 577 short sentences with simple structures, and the vocabulary set is made up of 32 POS tags, a subset of the 47 tags used for the WSJ. Due to the limited size of this corpus, ten sets of randomly partitioned train-test-held-out triples are generated to ensure the statistical significance of our results. We use 80 sentences for testing, 90 sentences for held-out data, and the rest for training. Before proceeding with the main discussion on training from the ATIS, we briefly describe the pretraining stage of the adaptive strategy.

\subsubsection{Pretraining with WSJ}

The idea behind the adaptive method is simply to make use of any existing labeled data. We hope that pretraining the grammars on these data might place them in a better position to learn from the new, sparsely labeled data. In the pretraining stage for this experiment, a grammar is directly induced from 3600 fully labeled WSJ sentences. Without any further training on ATIS data, this grammar achieves a parsing score of $87.3 \%$ on ATIS test sentences. The relatively high parsing score suggests that pretraining with WSJ has successfully placed the grammar in a good position to begin training with the ATIS data.

\subsubsection{Partially Supervised Training on ATIS}

We now return to the main focus of this experiment: learning from sparsely annotated ATIS training data. To verify whether some constituent classes are more informative than others, we could compare the parsing scores of the grammars trained using different constituent class labels. But this evaluation method does not take into account that the distribution of the constituent classes is not uniform. To normalize for this inequity, we compare the parsing scores to a baseline that characterizes the relationship between the performance of the trained grammar and the number of bracketed constituents in the training data. To generate the baseline, we create training data in which $0 \%$, $25 \%, 50 \%, 75 \%$, and $100 \%$ of the constituent brackets are randomly chosen to be included. One class of linguistic labels is better than another if its resulting parsing improvement over the baseline is higher than that of the other.

The test results of the grammars induced from these different training data are summarized in Figure 1. Graph (a) plots the outcome of using the direct induction strategy, and graph (b) plots the outcome of the adaptive strategy. In each graph, the baseline of random constituent brackets is shown as a solid line. Scores of grammars trained from constituent type specific data sets are plotted as labeled dots. The dotted horizontal line in graph (b) indicates the ATIS parsing score of the grammar trained on WSJ alone.

Comparing the five constituent types, we see that the HighP class is the most informative 


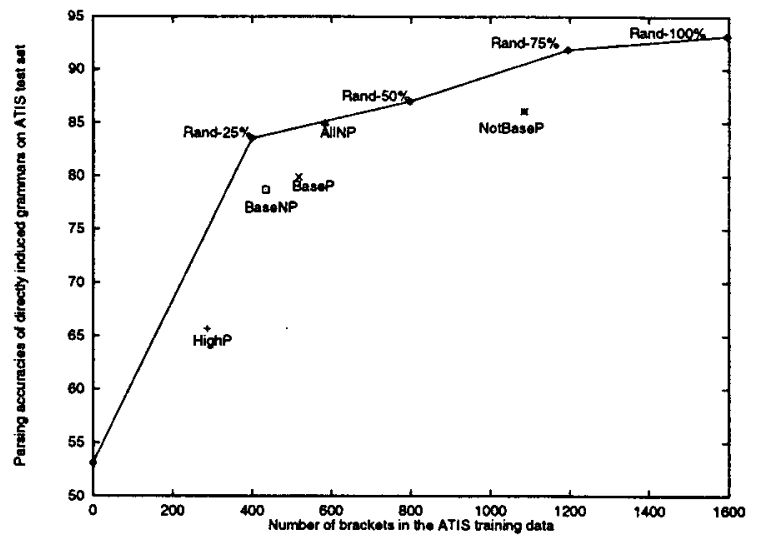

(a)

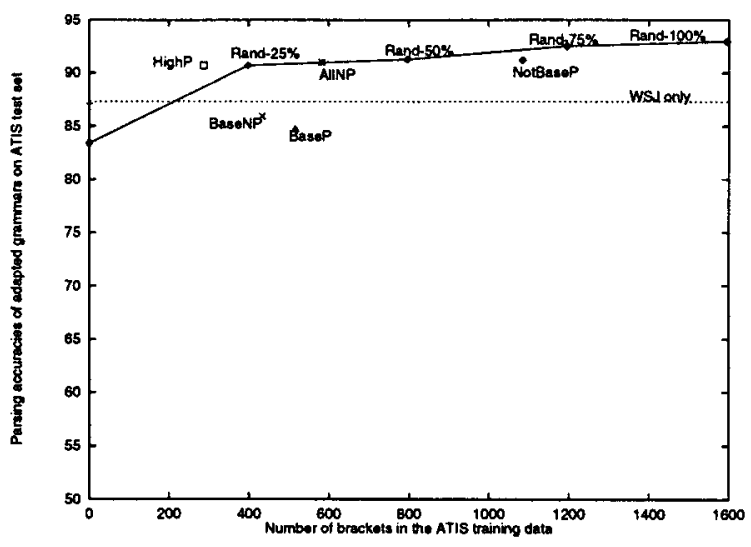

(b)

Figure 1: Parsing accuracies of (a) directly induced grammars and (b) adapted grammars as a function of the number of brackets present in the training corpus. There are 1595 brackets in the training corpus all together.

for the adaptive strategy, resulting in a grammar that scored better than the baseline. The grammars trained on the AllNP annotation performed as well as the baseline for both strategies. Grammars trained under all the other training conditions scored below the baseline. Our results suggest that while an ideal training condition would include annotations of both higher-level phrases and simple phrases, complex clauses are more informative. This interpretation explains the large gap between the parsing scores of the directly induced grammar and the adapted grammar trained on the same HighP data. The directly induced grammar performed poorly because it has never seen a labeled example of simple phrases. In contrast, the adapted grammar was already exposed to labeled WSJ simple phrases, so that it successfully adapted to the new corpus from annotated examples of higher-level phrases. On the other hand, training the adapted grammar on annotated ATIS simple phrases is not successful even though it has seen examples of WSJ higherlevel phrases. This also explains why grammars trained on the conglomerate class NotBaseP performed on the same level as those trained on the AllNP class. Although the NotBaseP set contains the most brackets, most of the brackets are irrelevant to the training process, as they are neither higher-level phrases nor simple phrases.

Our experiment also indicates that induction strategies exhibit different learning characteristics under partially supervised training conditions. A side by side comparison of Figure 1 (a) and (b) shows that the adapted grammars perform significantly better than the directly induced grammars as the level of supervision decreases. This supports our hypothesis that pretraining on a different corpus can place the grammar in a good initial search space for learning the new domain. Unfortunately, a good initial state does not obviate the need for supervised training. We see from Figure 1(b) that retraining with unlabeled ATIS sentences actually lowers the grammar's parsing accuracy.

\subsection{Experiment 2: Learning WSJ}

In the previous section, we have seen that annotations of complex clauses are the most helpful for inducing ATIS-style grammars. One of the goals of this experiment is to verify whether the result also holds for the WSJ corpus, which is structurally very different from ATIS. The WSJ corpus uses 47 POS tags, and its sentences are longer and have more embedded clauses.

As in the previous experiment, we construct training sets with annotations of different constituent types and of different numbers of randomly chosen labels. Each training set consists of 3600 sentences, and 1780 sentences are used as held-out data. The trained grammars are tested on a set of 2245 sentences.

Figure 2 (a) and (b) summarize the outcomes 


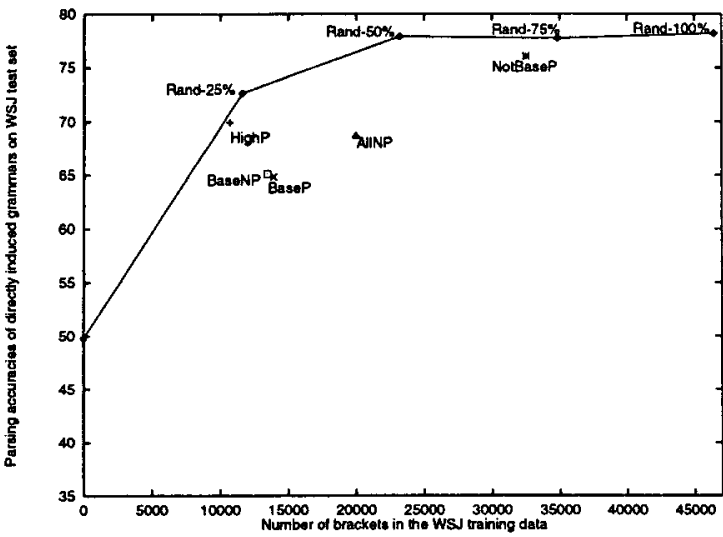

(a)

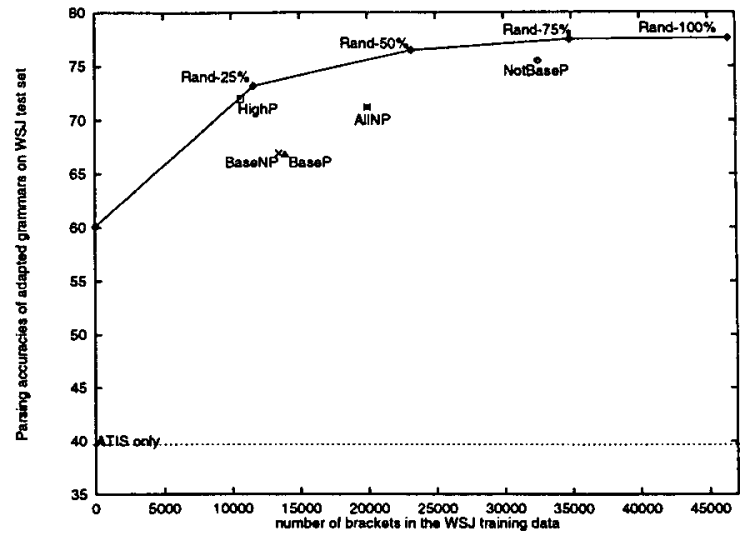

(b)

Figure 2: Parsing accuracies of (a) directly induced grammars and (b) adapted grammars as a function of the number of brackets present in the training corpus. There is a total of 46463 brackets in the training corpus.

of this experiment. Many results of this section are similar to the ATIS experiment. Higherlevel phrases still provide the most information; the grammars trained on the HighP labels are the only ones that scored as well as the baseline. Labels of simple phrases still seem the least informative; scores of grammars trained on BaseP and BaseNP remained far below the baseline. Different from the previous experiment, however, the AllNP training sets do not seem to provide as much information for this learning task. This may be due to the increase in the sentence complexity of the WSJ, which further de-emphasized the role of the simple phrases. Thus, grammars trained on AllNP labels have comparable parsing scores to those trained on HighP labels. Also, we do not see as big a gap between the scores of the two induction strategies in the HighP case because the adapted grammar's advantage of having seen annotated ATIS base nouns is reduced. Nonetheless, the adapted grammars still perform $2 \%$ better than the directly induced grammars, and this improvement is statistically significant. ${ }^{2}$

Furthermore, grammars trained on NotBaseP do not fall as far below the baseline and have higher parsing scores than those trained on HighP and AllNP. This suggests that for more complex domains, other linguistic constituents

\footnotetext{
${ }^{2} \mathrm{~A}$ pair-wise t-test comparing the parsing scores of the ten test sets for the two strategies shows $99 \%$ confidence in the difference.
}

such as verb phrases ${ }^{3}$ become more informative.

A second goal of this experiment is to test the adaptive strategy under more stringent conditions. In the previous experiment, a WSJ-style grammar was retrained for the simpler ATIS corpus. Now, we reverse the roles of the corpora to see whether the adaptive strategy still offers any advantage over direct induction.

In the adaptive method's pretraining stage, a grammar is induced from 400 fully labeled ATIS sentences. Testing this ATIS-style grammar on the WSJ test set without further training renders a parsing accuracy of $40 \%$. The low score suggests that fully labeled ATIS data does not teach the grammar as much about the structure of WSJ. Nonetheless, the adaptive strategy proves to be beneficial for learning WSJ from sparsely labeled training sets. The adapted grammars out-perform the directly induced grammars when more than $50 \%$ of the brackets are missing from the training data. The most significant difference is when the training data contains no label information at all. The adapted grammar parses with $60.1 \%$ accuracy whereas the directly induced grammar parses with $49.8 \%$ accuracy.

\footnotetext{
${ }^{3}$ We have not experimented with training sets containing only verb phrases labels (i.e., setting a pair of bracket around the head verb and its modifiers). They are a subset of the NotBaseP class.
} 


\section{Conclusion and Future Work}

In this study, we have shown that the structure of a grammar can be reliably learned without having fully specified constituent information in the training sentences and that the most informative constituents of a sentence are higherlevel phrases, which make up only a small percentage of the total number of constituents. Moreover, we observe that grammar adaptation works particularly well with this type of sparse but informative training data. An adapted grammar consistently outperforms a directly induced grammar even when adapting from a simpler corpus to a more complex one.

These results point us to three future directions. First, that the labels for some constituents are more informative than others implies that sentences containing more of these informative constituents make better training examples. It may be beneficial to estimate the informational content of potential training (unmarked) sentences. The training set should only include sentences that are predicted to have high information values. Filtering out unhelpful sentences from the training set reduces unnecessary work for the human annotators. Second, although our experiments show that a sparsely labeled training set is more of an obstacle for the direct induction approach than for the grammar adaptation approach, the direct induction strategy might also benefit from a two stage learning process similar to that used for grammar adaptation. Instead of training on a different corpus in each stage, the grammar can be trained on a small but fully labeled portion of the corpus in its first stage and the sparsely labeled portion in the second stage. Finally, higher-level constituents have proved to be the most informative linguistic units. To relieve humans from labeling any training data, we should consider using partial parsers that can automatically detect complex nouns and sentential clauses.

\section{References}

J.K. Baker. 1979. Trainable grammars for speech recognition. In Proceedings of the Spring Conference of the Acoustical Society of America, pages 547-550, Boston, MA, June.

E.J. Briscoe and N. Waegner. 1992. Robust stochastic parsing using the inside-outside algorithm. In Proceedings of the AAAI Work- shop on Probabilistically-Based NLP Techniques, pages 39-53.

E. Charniak. 1996. Tree-bank grammars. In Proceedings of the Thirteenth National Conference on Artificial Intelligence, pages 10311036.

E. Mark Gold. 1967. Language identification in the limit. Information Control, 10(5):447474.

C.T. Hemphill, J.J. Godfrey, and G.R. Doddington. 1990. The ATIS spoken language systems pilot corpus. In DARPA Speech and Natural Language Workshop, Hidden Valley, Pennsylvania, June. Morgan Kaufmann.

R. Hwa. 1998a. An empirical evaluation of probabilistic lexicalized tree insertion grammars. In Proceedings of COLING-ACL, volume 1, pages 557-563.

R. Hwa. 1998b. An empirical evaluation of probabilistic lexicalized tree insertion grammars. Technical Report 06-98, Harvard University. Available as cmp-lg/9808001.

K. Lari and S.J. Young. 1990. The estimation of stochastic context-free grammars using the inside-outside algorithm. Computer Speech and Language, 4:35-56.

M. Marcus, B. Santorini, and M. Marcinkiewicz. 1993. Building a large annontated corpus of english: the penn treebank. Computational Linguistics, 19(2):313-330.

F. Pereira and Y. Schabes. 1992. InsideOutside reestimation from partially bracketed corpora. In Proceedings of the 30th Annual Meeting of the $A C L$, pages 128-135, Newark, Delaware.

Y. Schabes and R. Waters. 1993. Stochastic lexicalized context-free grammar. In Proceedings of the Third International Workshop on Parsing Technologies, pages 257-266.

Y. Schabes, M. Roth, and R. Osborne. 1993. Parsing the Wall Street Journal with the Inside-Outside algorithm. In Proceedings of the Sixth Conference of the European Chapter of the $A C L$, pages 341-347. 